\title{
Experimental Evidence for Quantum Interference and Vibrationally Induced Decoherence in Single-Molecule Junctions
}

\author{
Stefan Ballmann, ${ }^{1}$ Rainer Härtle, ${ }^{2}$ Pedro B. Coto, ${ }^{2, *}$ Mark Elbing, ${ }^{3}$ Marcel Mayor, ${ }^{3,4}$ Martin R. Bryce, ${ }^{5}$ \\ Michael Thoss, ${ }^{2, \dagger}$ and Heiko B. Weber ${ }^{1, \dagger, \ddagger}$ \\ ${ }^{1}$ Lehrstuhl für Angewandte Physik, Friedrich-Alexander-Universität Erlangen-Nürnberg, Staudtstrasse 7, 91058 Erlangen, Germany \\ ${ }^{2}$ Institut für Theoretische Physik, Friedrich-Alexander-Universität Erlangen-Nürnberg, Staudtstrasse 7, 91058 Erlangen, Germany \\ ${ }^{3}$ Institute of Nanotechnology, Karlsruhe Institute of Technology (KIT), P.O. Box 3640, 76021 Karlsruhe, Germany \\ ${ }^{4}$ Department of Chemistry, University of Basel, St. Johanns-Ring 19, 4056 Basel, Switzerland \\ ${ }^{5}$ Department of Chemistry, Durham University, Durham, DH1 3LE, United Kingdom
}

(Received 19 March 2012; published 30 July 2012)

\begin{abstract}
We analyze quantum interference and decoherence effects in single-molecule junctions both experimentally and theoretically by means of the mechanically controlled break junction technique and densityfunctional theory. We consider the case where interference is provided by overlapping quasidegenerate states. Decoherence mechanisms arising from electronic-vibrational coupling strongly affect the electrical current flowing through a single-molecule contact and can be controlled by temperature variation. Our findings underline the universal relevance of vibrations for understanding charge transport through molecular junctions.
\end{abstract}

DOI: 10.1103/PhysRevLett.109.056801

PACS numbers: 85.65.+h, 72.10.Di, 73.23.-b, 73.40.Gk

Charge transport through single-molecule junctions $[1,2]$ is a quantum mechanical process. It was first treated as a purely electronic problem, combining transport concepts and quantum chemical calculations. Similarly, early experiments focused on the correlation between chemical structure and transport properties [3-6]. Inspired by the related process of electron transfer in molecules, which is strongly dominated by vibration-assisted electronic phenomena, it became evident that a purely electronic picture is also inadequate in single-molecule transport [7-9]. Vibrations may appear as vibrational side peaks to electronic transitions [10-14] and constitute a source for nonlinear phenomena [15,16] and bistabilities [17,18]. In addition, vibrations may also provide a strong decoherence mechanism in molecular junctions where electron transport is governed by destructive interference effects [19].

In a purely electronic picture, destructive interference is a built-in property of many molecules commonly used in molecular junctions [20-25]. It occurs when the current is carried by quasidegenerate electronic states (QDSs) which, e.g., differ in their spatial symmetry [see Fig. 1(a)]. In analogy to a double-slit experiment, these states provide different pathways (which are not necessarily spatially separated) for the electrons to tunnel through the molecular junction. Although their individual contribution to the current would be substantial, their phase-correct sum current can be very small [if the broadened levels overlap sufficiently; cf. Fig. 1(a)].

Interaction with vibrations may change this result significantly [19]. As a rule, electronic states couple very specifically to vibrations; in particular, a different electronic-vibrational coupling of the QDSs is expected. Consequently, destructive interference between the in- volved pathways is quenched, which affects the electron transmission, i.e., the electrical current. In more general terms, which-path information is provided by coupling to the vibrations.

In an experiment, vibrations are inherent to any molecular junction, in particular, at room temperature. Their influence can be controlled by systematically varying the temperature of the junction. When starting at low temperatures $T$, the influence of vibrations is small. With increasing $T$, decoherence will continuously be amplified via enhanced vibrational excitation. Hence, for systems which exhibit destructive interference, the expectation is an increase of the electrical current with increasing $T$ [cf. Figure 1(b)].
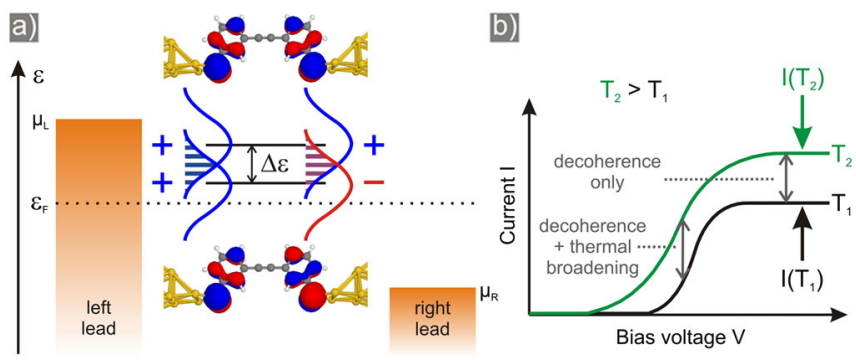

FIG. 1 (color online). (a) Schematic representation of a singlemolecule junction with two QDSs that are close in energy $(\Delta \epsilon)$ and different in their spatial symmetry. (b) In the resonant tunneling model including vibrationally induced decoherence [19], the current plateaus $I(T)$ are sensitive to vibrational excitation, which can be tuned by temperature. $I(T)$ is therefore a suitable observable to investigate vibrationally induced decoherence. 
The scope of this Letter is to analyze vibrationally induced decoherence in single-molecule junctions experimentally, accompanied by a detailed analysis of the molecular systems using density-functional theory. We have selected several molecules out of a class of rather typical molecular wires (conjugated, thiol or pyridine end capped) $[5,26,27]$. The employed systems are depicted in Fig. 2. In contrast to the molecule in the previous theoretical studies [19], which had a built-in $L \leftrightarrow R$ symmetry, we used molecules with a reduced symmetry: in particular, molecule 2 has two different end groups in meta and para position (cf. Ref. [28]). In molecule 3, the conjugated system is intersected in the center by a $\sigma$ bond, and the left and right conjugated subunits are spatially twisted by approximately $75^{\circ}$ with respect to each other (leading to electronic states localized either on the right or on the left end of the molecular rod). The study of systems 1-3 shows that $L \leftrightarrow R$ symmetric molecules are only a special case of a more general class of systems, where QDSs close to the Fermi energy generate vibration-sensitive interferences. For a specific molecule, the existence of such states also depends on the molecule-lead linker group. For example, molecule $\mathbf{4}$ has the full $L \leftrightarrow R$ symmetry. However, due to its pyridine anchor groups, the resulting QDSs are located remotely from the Fermi energy and do not contribute to the current. Therefore, it provides a counterexample where the considered mechanism is not active.

We use the mechanically controlled break junction technique at cryogenic vacuum conditions to establish singlemolecule junctions at atomically sharp, freestanding gold tips, and record current-voltage characteristics ( $I-V$ 's) $[3,29]$. We restricted our investigation to a temperature range of 8 to $40 \mathrm{~K}$, where the geometric structure of the junction is stable under temperature sweeps (see below). Above $40 \mathrm{~K}$, the junctions exhibit hysteresis and/or irreversibilities of the $I-V$ 's.

Typical $I-V$ curves are sketched in Fig. 1(b). The current flowing through a molecular junction is suppressed, as long as the voltage $V$ is sufficiently low that the electronic levels

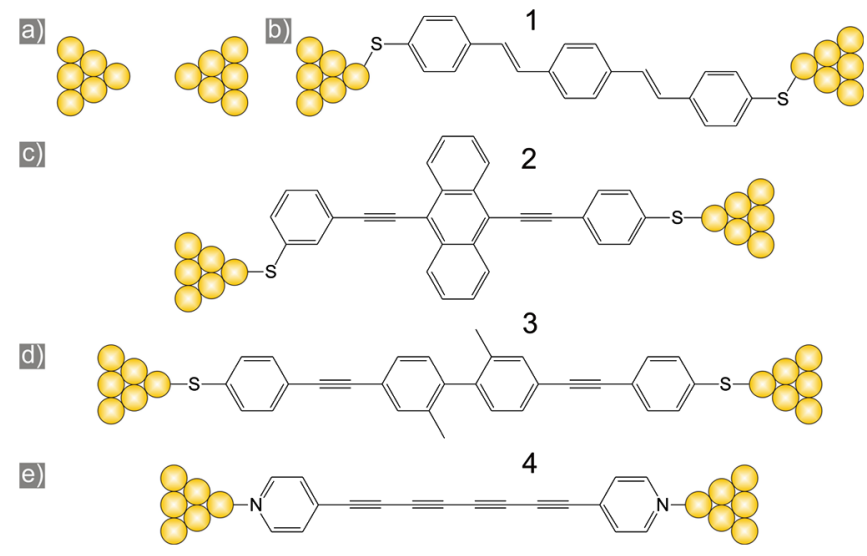

FIG. 2 (color online). Overview of the molecular systems investigated. of the junction are located outside the energy window defined by the two chemical potentials $\mu_{L}$ and $\mu_{R}$ in the left and the right leads, respectively $\left[V=\left(\mu_{L}-\mu_{R}\right) / e\right]$. This range of bias voltages defines the nonresonant transport regime. If one or more electronic levels are located within the bias window $\left[\mu_{L}, \mu_{R}\right]$, resonant transport processes occur. The transition is indicated by a steplike increase of the current level that remains almost constant for larger voltages unless another electronic state enters the bias window. This plateau often has a slightly positive slope, as quasicontinously new vibrational side channels are added, which can hardly be resolved [14]. It is on this first plateau in $I(V)$ where the analysis of the temperature dependence of the current level is carried out. It is particularly suited because in the resonant tunneling model (without vibrations) it is not affected by thermal broadening, i.e., the temperature dependence of the Fermi distribution function in the leads.

The temperature dependence of the electrical current flowing through a single-molecule junction has been considered before [7,30-33]. However, the experimental phenomenology presented in these papers is different and the previous discussion focused on effects that occur at much larger temperatures $(\gtrsim 150 \mathrm{~K})$ and/or in the nonresonant transport regime.

First, we present data on a blind experiment using a pair of gold electrodes without molecules. The question is whether the setup is sufficiently stable with respect to temperature variations. A temperature sweep from 10 to $33 \mathrm{~K}$ does not show significant variations of the tunneling current at low bias $[\leq 0.2 \mathrm{~V}$; see Figs. 3(a) and 3(b)]. At higher bias, though, irreversible drifts are observed at $T>22 \mathrm{~K}$. This small instability presumably occurs due to atomic rearrangements at high electric fields at the tips. This behavior is quite common in the absence of a bridging molecule, but we learned from many experiments that a bridging molecule stabilizes the junction and allows for stable conditions up to $\approx 1.5 \mathrm{~V}$ at low $T$ [4]. We conclude that in the temperature range considered the electrode pair provides a stable distance.

We now investigate the temperature dependence of the current flowing through a single-molecule junction. We first present data obtained with molecule 1 [see Fig. 3(c)]. The $I-V$ characteristics show a typical behavior: a fairly symmetric blockade region up to $\pm 0.43 \mathrm{~V}$ at $11 \mathrm{~K}$ and subsequently a steplike transition to a current plateau which, however, is less symmetric. When measuring the very same junction at $T=30 \mathrm{~K}$, two changes are evident. First, the blockade region is slightly reduced to $\pm 0.35 \mathrm{~V}$. Second, the current level taken at the first plateau of the $I-V$ characteristics is significantly increased. This becomes evident in Fig. 3(d), where the current level at $\pm 1.0 \mathrm{~V}$ [cf. arrows in Fig. 3(c)] is plotted versus temperature. In contrast to the blind experiment, a clear increase of the current by about $65 \%$ is observed, which remains reversible up to 

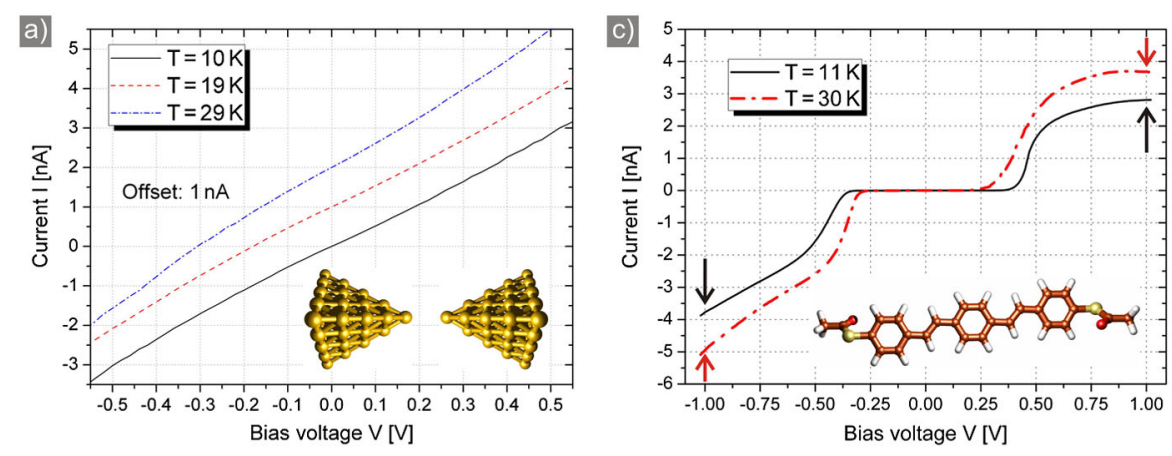

e)
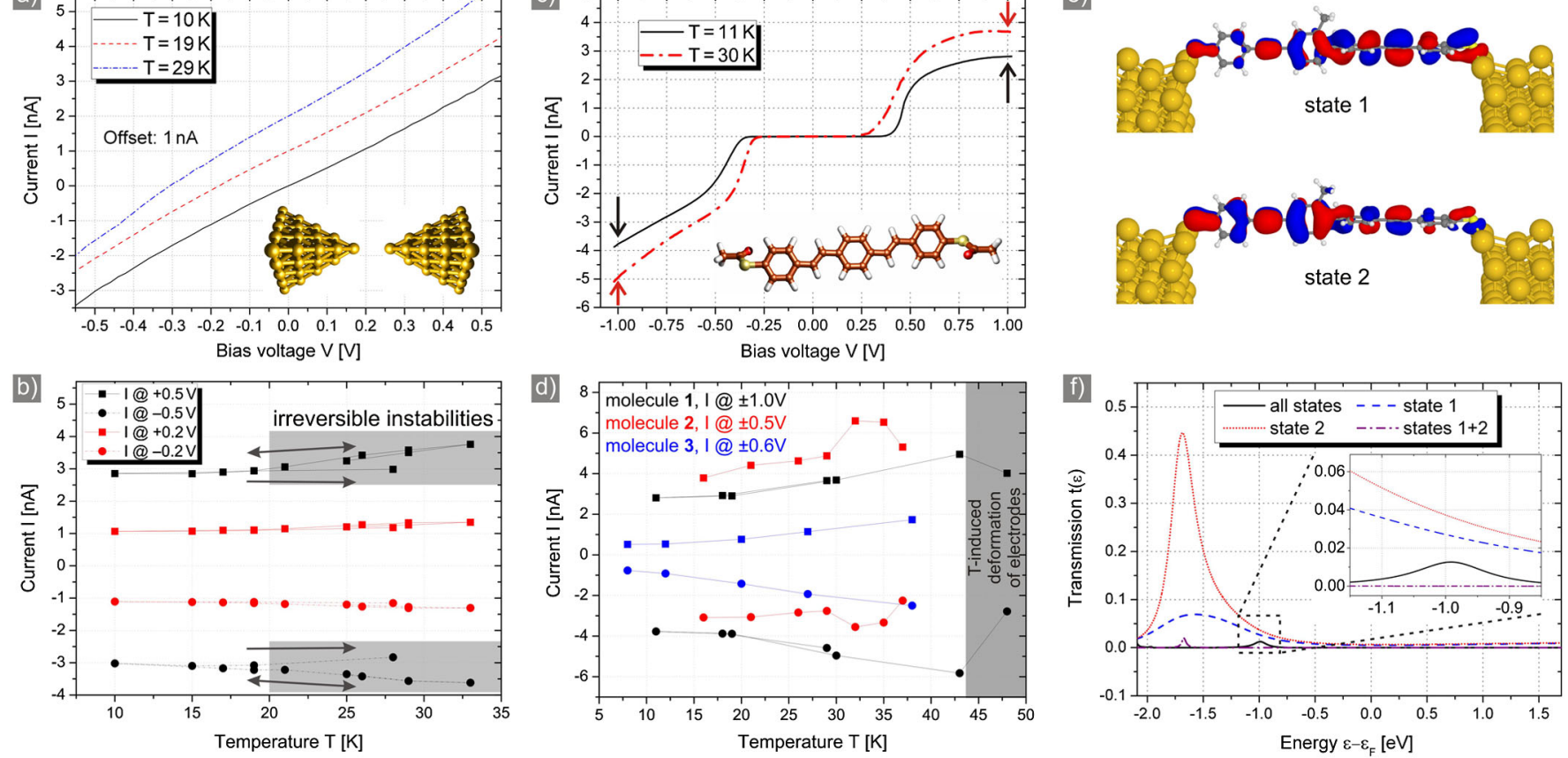

FIG. 3 (color online). $I-V$ and $I-T$ characteristics (a),(b) for gold electrodes (blind experiment), (c) for molecule 1, and (d) for molecules 1-3, respectively. The temperature-dependent current $I(T)$ recorded at the first plateau is the key observation indicating vibrationally induced decoherence. (e) Molecular junction 3 provides a pair of QDSs located next to the Fermi level. The corresponding wave functions differ in symmetry. (f) Transmission functions for electron transport through molecular junction 3 . The individual (broadened) peaks associated with the QDSs 1 and 2 at $\epsilon-\epsilon_{\mathrm{F}} \simeq-1.7 \mathrm{eV}$ are relatively strong, but their phase-correct transmission (states $1+2$ ) shows only a very small peak at this energy due to destructive interference. When including all states, this peak is shifted to $-1.0 \mathrm{eV}$ below $\epsilon_{\mathrm{F}}$.

$T \approx 40 \mathrm{~K}$. Again, at higher temperatures, we observe a drift. This phenomenology is qualitatively reproduced for several samples.

We continue by seeking more examples for this effect and investigate molecules $\mathbf{2}$ and $\mathbf{3}$. For all thiol end capped molecular wires (1-3), we observe qualitatively and reproducibly the same effect [see Fig. 3(d)]: an increase of the current with temperature that is intrinsic to the molecular junction, as inferred by the blind experiment.

The observed current increase $I(T)$ is too large to be explained by the well known vibrationally assisted tunneling effects. To understand the underlying mechanism, we analyze theoretically the transmission of molecular junction 3, which shows the most pronounced temperature effect in the experiment. For the clarity of the argument, we do not present the full theoretical treatment, as in [19], but restrict ourselves to a discussion of the respective transmission function. The transmission function is obtained by employing a model based on first-principles electronic structure calculations [19,34] within Landauer theory [1]. The result is depicted by the solid black line in Fig. 3(f). Peaks in this transmission function indicate that electrons can tunnel resonantly through the junction. Accordingly, the first plateau in the current is related to the peak closest to the Fermi level $\epsilon_{\mathrm{F}}$. For our example, this is the small peak at $\epsilon-\epsilon_{\mathrm{F}} \cong-1 \mathrm{eV}$ (see the inset).
This peak results predominantly from electron transport processes through two electronic states. The corresponding orbitals are shown in Fig. 3(e). They represent symmetric and antisymmetric combinations of molecular orbitals localized in the left and the right parts of the molecular bridge. If we take into account electron transport through just one of these states, we obtain transmission functions, which are depicted by the dotted red and dashed blue lines in Fig. 3(f). Both states are located relatively close in energy $(\Delta \epsilon \sim 0.02 \mathrm{eV})$ and are strongly broadened $(\sim 0.5 \mathrm{eV})$. This is what we termed QDSs [cf. Fig. 1(a)]. The broadening is a result of the strong coupling between the molecular bridge and the leads mediated by the sulfur end groups. The results show that electron transport through these states is governed by pronounced destructive quantum interference effects [19], which reduce their relatively large individual transmission function to a small peak at $\epsilon-\epsilon_{\mathrm{F}} \cong-1 \mathrm{eV}$. Note that the occurrence of QDSs is quite general and can be found in many molecular wires, irrespective of $L \leftrightarrow R$ symmetry.

Electronic-vibrational coupling can strongly quench such interference effects [19], leading to substantially larger electrical currents. This quenching is enhanced the more strongly the vibrational degrees of freedom are excited. A normal mode analysis of molecular junction 3 shows a broad distribution of vibrational modes located 
on the molecular bridge. Their frequencies range from $1.2 \mathrm{meV}$ to $0.4 \mathrm{eV}$, including 10 modes with a frequency lower than $10 \mathrm{meV}$ (approximately $2 k_{\mathrm{B}} T_{\max }$ ). Among these modes is the torsion $(3.7 \mathrm{meV})$ around the central $\sigma$ bond which is particularly important for the coupling of the left and right conjugated subunit, forming states 1 and 2 in Fig. 3(e). Increasing the temperature in the leads from $8 \mathrm{~K}$ to $T_{\max }=40 \mathrm{~K}$ leads to a substantial excitation of these modes and, via the thus enhanced vibrationally induced decoherence, to a larger current level. This correlates well with the experimental findings [see Fig. 3(d)].

In order to test the correlation between a temperature dependence of the first current plateau and the appearance of QDSs, we sought a counterexample. As the observed effect is robust even for asymmetric end groups, we selected a completely different system: molecular wire 4, which involves pyridine- instead of thiol-anchoring groups. The corresponding transmission function of this junction, which is depicted by the solid black line in Fig. 4(a), shows a number of peaks close to the Fermi level. Each of these peaks can be associated with a single, nondegenerate electronic state of this junction. This can be inferred by comparison to the dotted red, dashed blue, and dash-dotted gray lines representing the transmission probability for electron transport through individual states of the junction. This analysis shows that quantum interference effects and vibrationally induced decoherence are not expected to play a dominant role for electron transport through this junction.

The experimental results confirm this prediction: the temperature dependence of the first current plateau is indeed flat for the pyridine end capped wire $\mathbf{4}$, as displayed in Fig. 4(c). We conclude that the comparison of various molecules reveals a clear correlation: when QDSs are present, the first current plateau rises with temperature; if not, a temperature dependence is absent. This gives convincing evidence that decoherence due to coupling to vibrations plays an important role in single-molecule charge transport.
Another independent experimental strategy to test the relevance of decoherence is to modify the coupling to the leads via mechanically stretching the junction. We observed in the past that for thiol end capped molecules an Ångstrom-scale stretching of the molecular bridge often resulted in an increase of current, despite the fact that the opposite behavior is observed in empty tunnel junctions. We previously assumed that this modifies the transparency by altering the sulfur-gold bonding angle. With the given model at hand, another explanation occurs: when the coupling of the two QDSs to the leads is reduced, their mutual overlap becomes smaller and destructive interference effects are weakened. Experimentally, this would result in (i) a larger current level and (ii) a reduced temperature dependence.

We can test this conjecture by observing the temperature dependence upon stretching the junction. This was done experimentally for molecule $\mathbf{3}$ [displaying a $T$ dependence; see Fig. 4(b)] and 4 [displaying no $T$ dependence; see Fig. 4(c)]. For molecule 3, the current level increases upon stretching [in line with previous observations and prediction (i)] by a factor of 2-3. This can be interpreted as the reduction of destructive interference, but also different explanations may apply. It is instructive to analyze again the temperature dependence: for molecule $\mathbf{3}$, the relative increase of current, $\Delta I(T) / I$, is $230 \%$ in the given temperature range. This value reduces to $35 \%$ upon stretching, in line with (ii).

Again, the situation is different for molecule 4, which has no QDSs close to $\epsilon_{\mathrm{F}}$ : using the same protocol, the current level is slightly reduced upon stretching, since interference plays a minor role [see Fig. 4(c)]. The insensitivity to temperature, however, is maintained. The different response upon stretching gives additional support to the picture that resonant electron transport through the thiol end capped molecular junctions is governed by destructive interference.

We now revisit the irregular shape of the $I-T$ characteristics of molecule 2 . When a strong built-in asymmetry is
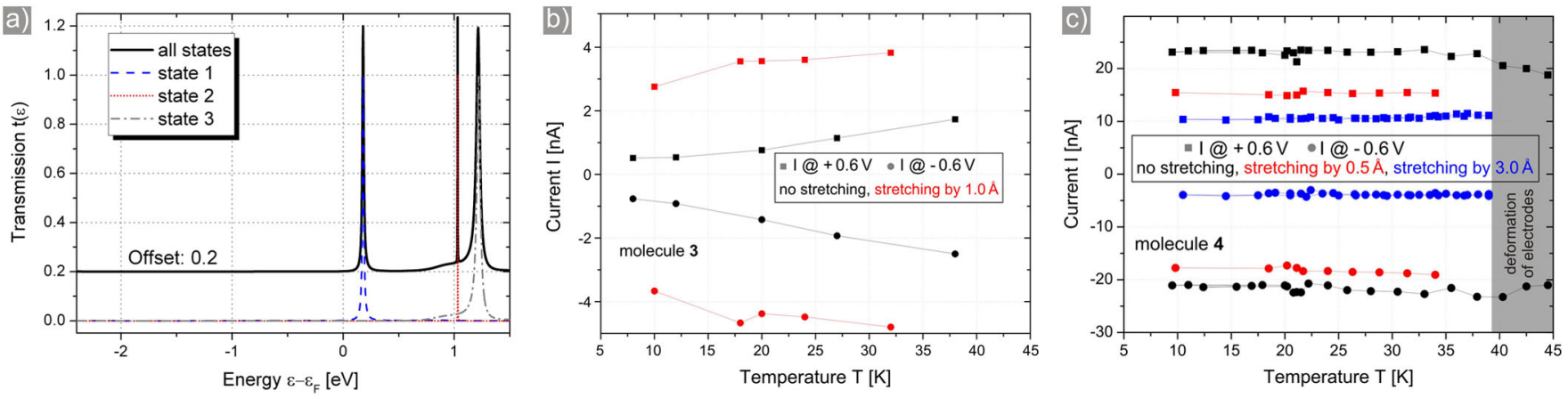

FIG. 4 (color online). (a) Transmission function for electronic transport through molecular junction 4 . The black curve is the superposition of the three individual contributions. In this molecular junction, the states close to the Fermi energy are not quasidegenerate and no destructive interference occurs. (b),(c) $I-V$ and $I-T$ characteristics upon stretching of molecular junctions 3 and $\mathbf{4}$, respectively. 
given, the current level is sensitive to charge reconfiguration at high bias $[5,16]$. This mechanism may also be affected by vibrations, and therefore additional temperature effects are not unexpected, in particular, for molecule $\mathbf{2}$ with its strong contact asymmetry.

To conclude, our studies reveal that the electrical current flowing through a single-molecule junction in the resonant transport regime exhibits an intrinsic temperature dependence. By analyzing four molecules experimentally and theoretically, this temperature effect can be correlated with the presence of QDSs, which give rise to destructive quantum interference. When increasing the temperature, the low-frequency modes of the junction are excited, which enhances decoherence and thus increases the current. We expect this phenomenon to be important for a broad class of molecular wires where QDSs are close to the Fermi energy, irrespective of symmetry conditions. Its impact on the transport characteristics will be significantly more pronounced at room temperature. The effect underscores that vibrations play a crucial and nontrivial role in electronic transport through molecular junctions.

The work was carried out in the framework of the Cluster of Excellence "Engineering of Advanced Materials" and the SFB 953 of the DFG. Support from the EC FP7 ITN FUNMOLS is gratefully acknowledged. Molecule 4 was synthesized by Dr. Changsheng Wang (Durham University).

*Present address: Instituto de Ciencia Molecular (ICMOL), Universitat de Valencia, Catedrático José Beltrán 2, 22085, E46071, Valencia, Spain.

${ }^{\dagger}$ Also at Interdisziplinäres Zentrum für Molekulare Materialien, Friedrich-Alexander-Universität ErlangenNürnberg, Staudtstrasse 7, 91058 Erlangen, Germany.

heiko.weber@physik.uni-erlangen.de

[1] J.C. Cuevas and E. Scheer, Molecular Electronics: An Introduction To Theory And Experiment (World Scientific, Singapore, 2010).

[2] N. A. Zimbovskaya and M. R. Pederson, Phys. Rep. 509, 1 (2011).

[3] J. Reichert, R. Ochs, D. Beckmann, H. B. Weber, M. Mayor, and H.v. Löhneysen, Phys. Rev. Lett. 88, 176804 (2002).

[4] J. Reichert, H. B. Weber, M. Mayor, and H. v. Löhneysen, Appl. Phys. Lett. 82, 4137 (2003).

[5] M. Elbing, R. Ochs, M. Koentopp, M. Fischer, C. von Hänisch, F. Weigend, F. Evers, H. B. Weber, and M. Mayor, Proc. Natl. Acad. Sci. U.S.A. 102, 8815 (2005).

[6] N. J. Tao, Nature Nanotech. 1, 173 (2006).

[7] A. Nitzan, Annu. Rev. Phys. Chem. 52, 681 (2001).

[8] W. Ho, J. Chem. Phys. 117, 11033 (2002).

[9] M. Galperin, M. A. Ratner, and A. Nitzan, J. Phys. Condens. Matter 19, 103201 (2007).
[10] R. Härtle, C. Benesch, and M. Thoss, Phys. Rev. B 77, 205314 (2008).

[11] R. Härtle, C. Benesch, and M. Thoss, Phys. Rev. Lett. 102, 146801 (2009).

[12] S. Ballmann, W. Hieringer, D. Secker, Q. Zheng, J. A. Gladysz, A. Görling, and H. B. Weber, Chem. Phys. Chem. 11, 2256 (2010).

[13] E. A. Osorio, K. O’Neill, N. Stuhr-Hansen, O. F. Nielsen, T. Bjørnholm, and H. S. J. van der Zant, Adv. Mater. 19, 281 (2007)

[14] D. Secker, S. Wagner, S. Ballmann, R. Härtle, M. Thoss, and H. B. Weber, Phys. Rev. Lett. 106, 136807 (2011).

[15] J. Koch and F. von Oppen, Phys. Rev. Lett. 94, 206804 (2005).

[16] R. Härtle and M. Thoss, Phys. Rev. B 83, 115414 (2011).

[17] A. S. Alexandrov and A. M. Bratkovsky, Phys. Rev. B 67, 235312 (2003).

[18] M. Galperin, M. A. Ratner, and A. Nitzan, Nano Lett. 5, 125 (2005).

[19] R. Härtle, M. Butzin, O. Rubio-Pons, and M. Thoss, Phys. Rev. Lett. 107, 046802 (2011).

[20] S. N. Yaliraki and M. A. Ratner, Ann. N.Y. Acad. Sci. 960, 153 (2002).

[21] G.C. Solomon, D.Q. Andrews, T. Hansen, R.H. Goldsmith, M. R. Wasielewski, R.P. Van Duyne, and M. A. Ratner, J. Chem. Phys. 129, 054701 (2008).

[22] D. M. Cardamone, C. A. Stafford, and S. Mazumdar, Nano Lett. 6, 2422 (2006).

[23] T. Markussen, R. Stadler, and K. S. Thygesen, Nano Lett. 10, 4260 (2010).

[24] T. A. Papadopoulos, I. M. Grace, and C. J. Lambert, Phys. Rev. B 74, 193306 (2006).

[25] C. M. Guédon, H. Valkenier, T. Markussen, K. S. Thygesen, J.C. Hummelen, and S.J. van der Molen, Nature Nanotech. 7, 305 (2012).

[26] A. Danilov, S. Kubatkin, S. Kafanov, P. Hedegård, N. Stuhr-Hansen, K. Moth-Poulsen, and T. Bjørnholm, Nano Lett. 8, 1 (2008).

[27] C. Wang, A. S. Batsanov, M. R. Bryce, S. Martín, R. J. Nichols, S. J. Higgins, V. M. García-Suárez, and C. J. Lambert, J. Am. Chem. Soc. 131, 15647 (2009).

[28] M. Mayor, H. B. Weber, J. Reichert, M. Elbing, C. von Hänisch, D. Beckmann, and M. Fischer, Angew. Chem., Int. Ed. Engl. 42, 5834 (2003).

[29] E. Lörtscher, H. B. Weber, and H. Riel, Phys. Rev. Lett. 98, 176807 (2007).

[30] Y. Selzer, M. A. Cabassi, T. S. Mayer, and D. L. Allara, J. Am. Chem. Soc. 126, 4052 (2004).

[31] M. Poot, E. Osorio, K. O’Neill, J. M. Thijssen, D. Vanmaekelbergh, C. A. van Walree, L.W. Jenneskens, and H. S. J. van der Zant, Nano Lett. 6, 1031 (2006).

[32] S. H. Choi, B. Kim, and C. D. Frisbie, Science 320, 1482 (2008).

[33] G. Sedghi et al., Nature Nanotech. 6, 517 (2011).

[34] C. Benesch, M. Čížek, J. Klimeš, I. Kondov, M. Thoss, and W. Domcke, J. Phys. Chem. C 112, 9880 (2008). 